\title{
An Interface Model Including Cracks and Roughness Applied to Masonry
}

\author{
Fazia Fouchal $^{\mathrm{a}}$, Frédéric Lebon ${ }^{\mathrm{b}, *}$, Maria L. Raffa ${ }^{\mathrm{b}, \mathrm{c}}$ and Giuseppe Vairo ${ }^{\mathrm{c}}$ \\ ${ }^{a}$ GEMH, Centre Universitaire de Génie Civil, Boulevard Jacques Derche, 19300 Egletons, France \\ ${ }^{b}$ LMA, Aix-Marseille University, CNRS, Centrale Marseille, 31 Chemin Joseph-Aiguier, F-13402 Marseille Cedex 20, \\ France \\ 'Department of Civil Engineering and Computer Science (DICII), University of Rome "Tor Vergata", via del \\ Politecnico 1, 00133 Rome, Italy
}

\begin{abstract}
In this paper an interface model accounting for roughness and micro-cracks is presented and applied to masonry-like structures. The model is consistently derived by coupling a homogenization approach and arguments of asymptotic analyses. A numerical procedure is introduced and numerical results, based on a finite element formulation, are successfully compared with experimental data, obtained on masonry samples undergoing to shear tests. Finally, a parametric numerical analysis is proposed, highlighting the influence of the roughness features on the interface response.
\end{abstract}

Keywords: Asymptotic analysis, masonry structures micro-cracks, roughness, soft interface.

\section{INTRODUCTION}

In the general framework of micro-analysis applied to model masonry structures, basically, two main approaches can be distinguished: the so-called simplified micromodeling approach and the detailed micro-modeling approach.

The first method consists of modeling mortar and unit/mortar interfaces as a unique discontinuous element, usually referred to as joint. In the last few decades, after the first attempt due to Page [1], several authors have focused this kind of micro-modeling approach and a number of models can be found in the specialized literature [2-7]. Lofti and Shing [2], Lourenço and Rots [4], Gambarotta and Lagomarsino [3] among others, have proposed interface models, which include concepts developed in the theory of plasticity for non-standard materials and in fracture mechanics. All these models adopt a Mohr-Coulomb failure criteria and use an internal variable to describe a post-peak softening behavior. These interface constitutive models are usually expressed in terms of contact tractions and conjugate generalized joint strains, derived from the discontinuities of the displacements at the joint. In particular, Lofti and Shing [2] presented a constitutive model for dilatant interfaces that was able to simulate initiation and propagation of fracture under combined normal and tangential stresses. Gambarotta and Lagomarsino [3] proposed a simplified micro-modeling

*Address correspondence to this author at the LMA, Aix-Marseille University, Cnrs, Centrale Marseille, 31 Chemin Joseph-Aiguier, F-13402 Marseille; Tel: +33(0)4 91164051; Fax: +33 (0)4 91164481;

E-mail: lebon@lma.cnrs-mrs.fr method based on continuum damage mechanics (CDM) which takes into account both the mortar damage and the unit-mortar decohesion caused by opening and frictional sliding. Raous et al. [5] have developed a model, improved by Monerie and Raous [8], known as RCCM model, which predicts the damage evolution at the interface among two initially-bonded deformable bodies. They proposed a consistent model that accounts for adhesion, Coulomb friction and unilateral contact. Adhesion and friction were strictly coupled with damage which was modelled in agreement with the Frémond theory [9]. This model was adopted by Fouchal et al. [10], to study interfaces in masonry assemblies.

In the detailed micro-modeling approach, the masonry joints are represented by mortar continuous elements and by discontinuous interface elements. This type of approach is probably less common in the literature because of its significant computational cost, but it has been proved as a powerful tool to simulate, in an accurate way, the behavior of small masonry assemblies [11-14]. Alfano and Sacco [11] proposed an interesting interface model, combining elastoplasticity arguments and CDM approach, introducing, within an interface representative element volume, the distinction between a linear elastic undamaged zone and a damaged zone, considering a unilateral Coulomb friction law. Pelissou and Lebon [12] applied model by Gambarotta and Lagomarsino for characterizing the local behavior of the brick/mortar interface, by coupling the asymptotic techniques (particularly, matched asymptotic expansions) [15-17]. 
Recently, Rekik and Lebon [13, 14] proposed a detailed micro-mechanical model based on homogenization theories, CDM and asymptotic techniques. The rationale of that model is based on the assumption that the non-linear behavior of masonry is concentrated at unit/mortar interface level. In order to estimate an interface constitutive law, they assume the existence of a third material, called interphase, which is a mixture of the two principal masonry constituents, brick and mortar indeed. In order to obtain the effective mechanical properties of such a micro-cracked brick/mortar interface, a three steps method is introduced. Firstly, a classical homogenization procedure for stratified composites is used in order to derive virgin material properties; then a homogenization technique of damage mechanics [18] is applied to take into account the presence of micro-cracks; finally, an asymptotic strategy leads to brick/mortar interface constitutive law.

In this paper a detailed micro-modeling approach is adopted. In this framework, a generalization of the RekikLebon model is proposed. The novelty of this paper is the introduction of an internal variable, called roughness function, which takes into account the geometrical heterogeneities localized at the unit/mortar interface level.

The paper is organized as follows. In Section 2, the general theoretical background is traced, by introducing the reference problem, and the interface model is developed within the context of the micro-mechanical analysis and of the asymptotic strategy. Then, Section 3 is devoted to the experimental results obtained by shear tests on small masonry assemblies [10, 19]. Finally, in Section 4, numerical results based on the proposed approach are presented and discussed. They show model soundness and consistency and are obtained via a finite element formulation that involves interface elements to model unit/mortar interfaces. The experimental data presented in Section 3 are used to set the numerical model, enabling to carry out a parametric analysis on the roughness features. In detail, after the identification of model parameters, the influence of both shape and entity of the roughness function on the global response is discussed.

\section{PROBLEM STATEMENT AND MODEL FORMULATION}

In the following, reference will be made to the model proposed by Rekik and Lebon [13, 14], aiming to furnish a generalization of that approach that includes a roughness characterization at the interface level. The proposed model fits into the general framework of the asymptotic expansions method.

This method was, originally, developed by SanchezPalencia [20] in order to derive the homogenized response of composites. It represents a mathematically rigorous way to recover the governing equations of the interface models and of the plate and shell theories. Basically, the method is based on the choice of a geometrically small parameter (e.g. the size of the microstructure or the thickness of an interphase layer) and on the expansion of the relevant fields (e.g. displacement, stress and strain) in power series with respect to the chosen small parameter. In the following, the matched asymptotic expansions method $[21,22]$, i.e. one-direction asymptotic expansions method with matching conditions, is used. Accordingly, the derivation of the governing equations of the soft interface is performed by adopting the strong formulation of the equilibrium problem, i.e. by writing the classical compatibility, constitutive and equilibrium equations. The matched asymptotic expansions method is chosen because of its efficiency in recovering an elastic interface law which retains memory of interphase material properties, as, in this case, roughness and micro-cracks.

In what follows, the interface model is discussed and an application on the unit/mortar interface of masonry structures is performed.

\subsection{Notation}

Let the Cartesian frame $\left(O, x_{1}, x_{2}, x_{3}\right)$ be introduced, and let $\left(i_{1}, i_{2}, i_{3}\right)$ be the corresponding orthonormal basis. With reference to the notation defined in Fig. (1), a thin layer $B^{\varepsilon}$ (called interphase) with non-constant small thickness $\eta \varepsilon$ is considered, the scale quantity $\varepsilon$ being such that $0<\varepsilon<<1$. The function $\eta$ will be defined in which follows. Moreover, let $S$ be the intersection between $B^{\mathcal{E}}$ and the interphase midplane, $S$, referred to as interface, being an open bounded set in $\mathbb{R}^{2}$ with a smooth boundary. Thus, the reference frame origin $O$ lies at the interphase midplane and the $x_{3}$ axis runs perpendicular to $S$. The interphase lies between two bodies, generally denoted as adherents, occupying the reference configuration $\Omega_{ \pm}^{\varepsilon} \subset \mathbb{R}^{3}$. In such a way, the interphase represents the adhesive joining the two bodies $\Omega_{+}^{\varepsilon}$ and $\Omega_{-}^{\varepsilon}$. It is assumed that the adhesive and the adherents are perfectly bonded, and therefore the continuity of displacements and stress vector fields is ensured. Let $S_{ \pm}^{\varepsilon}$ be the surfaces between the interphase and the adherents, and let $\Omega^{\varepsilon}=\Omega_{ \pm}^{\varepsilon} \cup S_{ \pm}^{\varepsilon} \cup B^{\varepsilon}$ be the overall system.

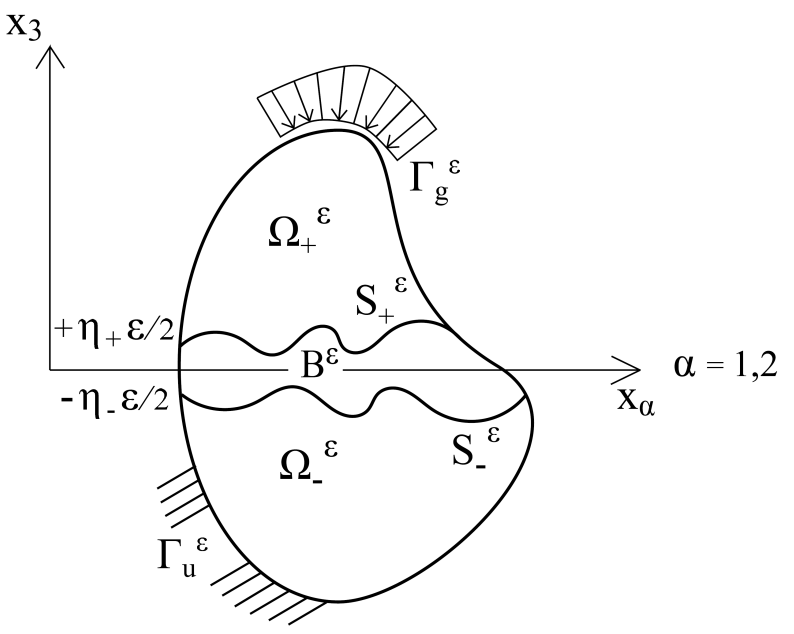

Fig. (1). Problem statement and notation.

As a definition, let $\eta_{ \pm}\left(x_{1}, x_{2}\right) \in C_{S, \mathbb{R}^{2}}^{0}$ be the roughness functions describing $S_{+}^{\varepsilon}$ and $S_{-}^{\varepsilon}$, respectively. They are assigned functions that take into account the geometrical 
shape at the adherents/interphase interface level, and they are assumed to satisfy the following properties:

$$
\left\{\begin{array}{l}
-\frac{\varepsilon}{2} \eta_{-}\left(x_{1}, x_{2}\right) \leq x_{3} \leq \frac{\varepsilon}{2} \eta_{+}\left(x_{1}, x_{2}\right) \\
\eta_{-}\left(x_{1}, x_{2}\right)>0 \\
\eta_{+}\left(x_{1}, x_{2}\right)>0
\end{array}\right.
$$

\section{Besides, let}

$$
\eta\left(x_{1}, x_{2}\right):=\frac{\eta_{-}\left(x_{1}, x_{2}\right)+\eta_{+}\left(x_{1}, x_{2}\right)}{2}
$$

be the average measure of the roughness function in the limit problem for $\varepsilon$ tending to zero (see Fig. 2).

Each subregion of such a composite system has been assumed to be homogeneous and materials are considered to behave as linearly elastic, with $\mathbf{a}_{ \pm}$and $\mathbf{b}^{\varepsilon}$ denoting the positive-definite fourth-order elasticity tensors of the adherents and the interphase, respectively. The adherents are subjected to the body force density $\mathbf{f}: \Omega_{ \pm}^{\varepsilon} \mapsto \mathbb{R}^{3}$ and to the surface density $\mathbf{g}: \Gamma_{g}^{\mathcal{E}} \mapsto \mathbb{R}^{3}$. Body forces are neglected in the adhesive region. On the boundary portion $\Gamma_{u}^{\varepsilon}$, homogeneous boundary conditions are prescribed:

$\mathbf{u}^{\varepsilon}=\mathbf{0} \quad$ on $\Gamma_{u}^{\varepsilon}$

where $\mathbf{u}^{\varepsilon}: \Omega_{ \pm}^{\varepsilon} \mapsto \mathbb{R}^{3}$ is the displacement field defined on $\Omega^{\varepsilon}$. Let the boundary parts $\Gamma_{g}^{\mathcal{E}}$ and $\Gamma_{u}^{\varepsilon}$ be located far from the interphase domain and let the fields representing the external forces be endowed with sufficient regularity, in order to ensure the existence of the equilibrium configuration.

\subsection{Rescaled problem}

A classical change of scale is performed both in adhesive and in adherents. In what follows, symbol $\left({ }^{\wedge}\right)$ denotes the rescaled fields in the adhesive and symbol ( $\left(^{-}\right)$the rescaled fields in the adherents. Accordingly, addressing Fig. (2), the following rules for the change of variables hold:

$$
\begin{aligned}
& \hat{\mathbf{p}}:\left(x_{1}, x_{2}, x_{3}\right) \rightarrow\left(z_{1}, z_{2}, z_{3}\right)=\left(x_{1}, x_{2}, x_{3} / \varepsilon\right) \\
& \overline{\mathbf{p}}:\left(x_{1}, x_{2}, x_{3}\right) \rightarrow\left(z_{1}, z_{2}, z_{3}\right)=\left(x_{1}, x_{2}, x_{3}+1 / 2 \mp \varepsilon / 2\right)
\end{aligned}
$$

where the plus (respectively, minus) sign applies whenever $x \in \Omega_{+}^{\varepsilon}$ (respectively, $x \in \Omega_{-}^{\varepsilon}$ ).

By enforcing relationships (2), the rescaled domains for interphase and adherents are described by:

$$
\begin{aligned}
& B=\left\{\left(z_{1}, z_{2}, z_{3}\right) \in \mathbb{R}^{3}:\left(z_{1}, z_{2}\right) \in S,-\frac{\eta_{-}}{2}<z_{3}<\frac{\eta_{+}}{2}\right\} \\
& \Omega_{ \pm}=\Omega_{ \pm}^{\varepsilon} \pm \frac{\eta_{ \pm}}{2}(1-\varepsilon) \mathbf{i}_{3}
\end{aligned}
$$

The sets $S_{ \pm}=\left\{\left(z_{1}, z_{2}, z_{3}\right) \in \mathbb{R}^{3}:\left(z_{1}, z_{2}\right) \in S, z_{3}= \pm \frac{\eta_{ \pm}}{2}\right\}$ denote the interfaces between $B$ and $\Omega_{ \pm}$, and $\Omega=\Omega_{+} \cup \Omega_{-} \cup B \cup S_{+} \cup S_{-} \quad$ denotes the rescaled configuration of the composite body (see Fig. 2). Finally, $\Gamma_{u}$ and $\Gamma_{g}$ are the images of $\Gamma_{u}^{\mathcal{E}}$ and $\Gamma_{g}^{\mathcal{E}}$ after the rescaling, and $\overline{\mathbf{f}}:=\mathbf{f} \circ \overline{\mathbf{p}}^{-\mathbf{1}}$ and $\overline{\mathbf{g}}:=\mathbf{g} \circ \overline{\mathbf{p}}^{-\mathbf{1}}$ are the rescaled volume force density and the rescaled surface force density, respectively.

Denoting with $\hat{\mathbf{u}}^{\varepsilon}:=\mathbf{u}^{\varepsilon} \circ \hat{\mathbf{p}}^{-\mathbf{1}}$ and $\overline{\mathbf{u}}^{\boldsymbol{\varepsilon}}:=\mathbf{u}^{\boldsymbol{\varepsilon}} \circ \overline{\mathbf{p}}^{-\mathbf{1}}$, the displacement fields relevant to the rescaled adhesive and adherents, respectively, the following asymptotic expansions up to the first order with respect to the small parameter $\varepsilon$ hold:

$$
\begin{aligned}
& \mathbf{u}^{\varepsilon}\left(z_{1}, z_{2}, z_{3}\right)=\mathbf{u}^{0}+\varepsilon \mathbf{u}^{1}+o(\varepsilon) \\
& \hat{\mathbf{u}}^{\varepsilon}\left(z_{1}, z_{2}, z_{3}\right)=\hat{\mathbf{u}}^{0}+\varepsilon \hat{\mathbf{u}}^{1}+o(\varepsilon) \\
& \overline{\mathbf{u}}^{\varepsilon}\left(z_{1}, z_{2}, z_{3}\right)=\overline{\mathbf{u}}^{0}+\varepsilon \overline{\mathbf{u}}^{1}+o(\varepsilon)
\end{aligned}
$$

The corresponding asymptotic expansions for the strain tensors, in interphase and in the adherents, respectively, result from:
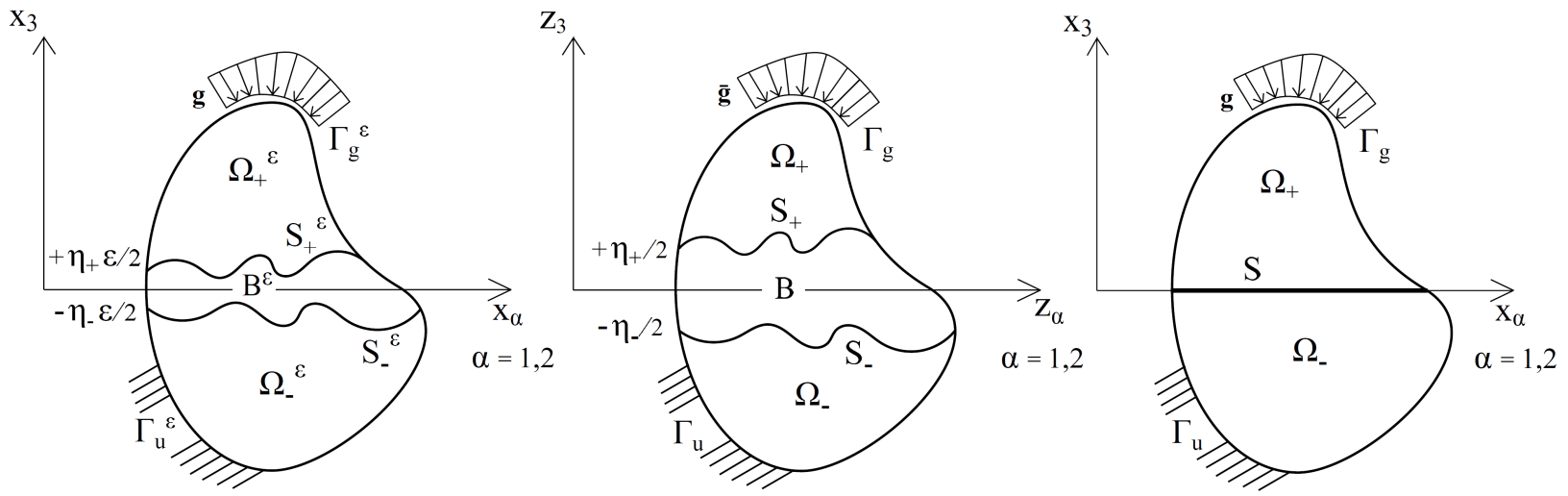

Fig. (2). Problem rescaling: (a) unscaled system, (b) rescaled system, (c) limit system. 


$$
\begin{aligned}
& \hat{\mathbf{e}}\left(\hat{\mathbf{u}}^{\varepsilon}\right)=\varepsilon^{-1} \hat{\mathbf{e}}^{-1}+\hat{\mathbf{e}}^{0}+O(\varepsilon) \\
& \overline{\mathbf{e}}\left(\overline{\mathbf{u}}^{\varepsilon}\right)=\varepsilon^{-1} \overline{\mathbf{e}}^{-1}+\overline{\mathbf{e}}^{0}+O(\varepsilon)
\end{aligned}
$$

with:

$\hat{\mathbf{e}}^{-1}=\left[\begin{array}{cc}0 & \frac{1}{2} \hat{u}_{\alpha, 3}^{0} \\ \frac{1}{2} \hat{u}_{\alpha, 3}^{0} & \hat{u}_{3,3}^{0}\end{array}\right]$

$\hat{\mathbf{e}}^{0}=\left[\begin{array}{cc}\operatorname{Sym}\left(\hat{u}_{\alpha, \beta}^{0}\right) & \frac{1}{2}\left(\hat{u}_{3, \alpha}^{0}+\hat{u}_{\alpha, 3}^{1}\right) \\ \frac{1}{2}\left(\hat{u}_{3, \alpha}^{0}+\hat{u}_{\alpha, 3}^{1}\right) & \hat{u}_{3,3}^{1}\end{array}\right]$

$\overline{\mathbf{e}}^{-1}=\mathbf{0}$

$\overline{\mathbf{e}}^{0}=\left[\begin{array}{cc}\operatorname{Sym}\left(\bar{u}_{\alpha, \beta}^{0}\right) & \frac{1}{2}\left(\bar{u}_{3, \alpha}^{0}+\bar{u}_{\alpha, 3}^{1}\right) \\ \frac{1}{2}\left(\bar{u}_{3, \alpha}^{0}+\bar{u}_{\alpha, 3}^{1}\right) & \bar{u}_{3,3}^{1}\end{array}\right]$

where, as it is customary, Greek indexes vary in the set $\{1,2\}$.

Denoting with $\hat{\boldsymbol{\sigma}}^{\varepsilon}:=\boldsymbol{\sigma}^{\varepsilon} \circ \hat{\mathbf{p}}^{-\mathbf{1}}$ and $\overline{\boldsymbol{\sigma}}^{\varepsilon}:=\boldsymbol{\sigma}^{\varepsilon} \circ \overline{\mathbf{p}}^{-\mathbf{1}}$, the stress fields from the rescaled adhesive and adherents, respectively, the following stress asymptotic expansions with respect $\varepsilon$ also hold:

$\boldsymbol{\sigma}^{\varepsilon}=\boldsymbol{\sigma}^{0}+o(\varepsilon)$

$\hat{\boldsymbol{\sigma}}^{\varepsilon}=\hat{\boldsymbol{\sigma}}^{0}+o(\varepsilon)$

$\overline{\boldsymbol{\sigma}}^{\varepsilon}=\overline{\boldsymbol{\sigma}}^{0}+o(\varepsilon)$

Since the representation form (13), the equilibrium equations of the interphase, addressed by neglecting the corresponding body forces, leads to:

$\hat{\sigma}_{i 3,3}^{0}=0$

that is, stress components $\hat{\sigma}_{i 3}^{0}$ (with $i \in\{1,2,3\}$ ) have to be described as constant with respect to $z_{3}$ in the adhesive. Moreover, by enforcing the continuity of the stress field among the adhesive and the adherents, Eq. (15) leads to:

$\left[\left[\sigma_{i 3}^{0}\right]\right]=0$

where, as a notation rule, $[[f]]:=f\left(x_{1}, x_{2}, 0^{+}\right)-f\left(x_{1}, x_{2}, 0^{-}\right)$ denotes the jump across the surface $S$ of a function $f$ defined on the limit configuration obtained for $\varepsilon \mapsto 0^{+}$(see Fig. 2).

\subsection{Interphase/interface analysis}

In the framework of the previously-introduced notation, the following constitutive laws hold for adherents and interphase, respectively, in the rescaled problem: $\overline{\boldsymbol{\sigma}}^{\varepsilon}=\mathbf{a}^{ \pm} \mathbf{e}\left(\overline{\mathbf{u}}^{\varepsilon}\right)$

$\hat{\boldsymbol{\sigma}}^{\varepsilon}=\mathbf{b}^{\varepsilon} \mathbf{e}\left(\hat{\mathbf{u}}^{\varepsilon}\right)$

By assuming that the interphase is described by a soft behavior $[21,22]$, the following condition can be stated:

$\mathbf{b}^{\varepsilon}=\mathbf{c b}+o(\varepsilon)$

In order to refer to a matrix notation, let $\left(K^{j l}\right)_{k i}:=b_{i j k l}$ be introduced. By substituting Eqs. (7) and (13) into Eq. (18), and integrating with respect to $z_{3}$, the following soft interface law is obtained:

$\hat{\boldsymbol{\sigma}}^{0} \mathbf{i}_{3}=\frac{1}{\eta} \mathbf{K}^{33}\left[\hat{\mathbf{u}}^{\mathbf{0}}\right]$

where the influence on the interface stiffness of the roughness function $\eta$ is clearly established.

Referring to the bi-dimensional problem in the plane $\left(i_{1}, i_{3}\right)$, for the sake of simplicity, the stiffness matrix $\mathbf{K}^{\mathbf{3 3}}$ in Eq. (20) is obtained starting from the micro-cracked material of Kachanov's theory [18] and it is expressed as follows:

$\mathbf{K}^{\mathbf{3 3}}=\left[\begin{array}{cc}K_{11}^{33} & K_{13}^{33} \\ K_{13}^{33} & K_{33}^{33}\end{array}\right]=\left[\begin{array}{cc}\frac{L}{B_{T} l^{2}} & 0 \\ 0 & \frac{L}{2 B_{N} l^{2}}\end{array}\right]$

in which $B_{T}$ and $B_{N}$ represent the components of the crack compliance tensor $\mathbf{B}$ introduced in [18] along the tangent (i.e., along $i_{1}$ ) and normal-to-the-interface (i.e., along $i_{3}$ ) directions, respectively, and $L$ is the characteristic dimension of the limit interface domain along $\mathbf{i}_{\mathbf{1}}$. Moreover, the interface stiffness matrix depends on the parameter $l$, that identifies an average overall measure of micro-cracks. In this model, following the approach proposed by Rekik and Lebon [14], a simplified bilinear micro-cracks evolution law is adopted by assuming a dependence of the parameter $l$ on the global shear stress at the interface.

\section{EXPERIMENTAL STUDY}

The experimental tests, which this paper refers to, have been carried by Fouchal [19]. The small masonry assemblies considered are made by two or three, hollow and full bricks, bound together by mortar joints. The mortar used in specimen is a conventional mortar ready-made based on a mixture of aggregates, rolled sand-lime and portland cement. In order to determine the mechanical properties of both materials, some bending and compressive tests were performed. The obtained results, discussed in more detail in [10], are summarized in Table $\mathbf{1}$.

\subsection{Shear Tests on Coupled and Triplet Samples}

Aiming to observe the shear behavior of the masonry assembly at the local level, several experimental tests, concerning samples composed by two [23], three or more 
bricks [24], are proposed in literature. The considered experimental tests concern some coupled and triplet samples, shown schematically in Fig. (3), subjected to simple shear load. In order to study the influence of brick type on the shear behavior, two different types of bricks, i.e. full and hollow, are used and, for both type of triplet, four tests are performed. More details can be found in $[10,19]$. In the following, reference will be made to experimental results on triplet samples only.

Table 1. Experimentally-determined mechanical properties of the three-fold masonry constituents.

\begin{tabular}{|l|c|}
\hline Young's moduli (MPa) of full brick & 9450 \\
\hline Poisson ratio of full brick & 0.13 \\
\hline Young's moduli (MPa) of hollow brick & 6050 \\
\hline Poisson ratio of hollow brick & 0.13 \\
\hline Young's moduli (MPa) of mortar & 8200 \\
\hline Poisson ratio of mortar & 0.3 \\
\hline
\end{tabular}

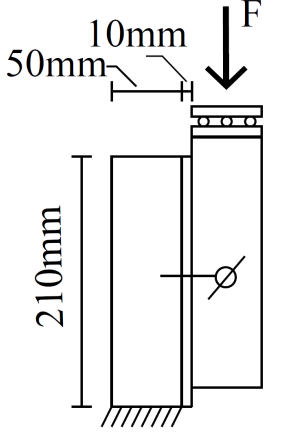

(a)

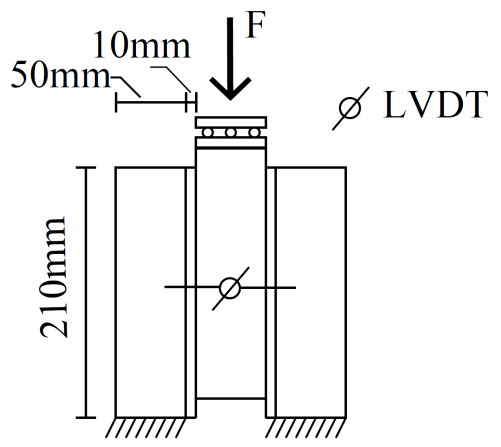

(b)
Fig. (3). Shear tests. (a) Two bricks sample. (b) Three bricks sample. LVDT: linear variable differential transformer.

The shear test consists of applying a monotonically increasing load $F$ on the top of the samples, until the failure (see Fig. 3). During the test, LVDT sensors fixed on the sample, give an average measure of the relative displacement between bricks. The obtained results, in terms of global shear stress and relative displacement, on the triplet samples of full and hollow bricks were shown, respectively, in Fig. (4) and Fig. (5). In the first case, the mechanical behavior is characterized by brittle behavior with an elastic part characterized by high values of the stiffness for test 1 and 4 and reduced values of the stiffness for the test 2 and 3. In the hollow bricks case, a softening behavior was observed with an initial elastic branch characterized by high values of stiffness. After reaching the maximum load value, the response was characterized by sliding motion between bricks $[10,19]$.

One remarks that the failure load value differs between the tests performed on similar samples. Fig. (6) underlines the dispersion of the failure modes that probably is the cause of the above cited difference in terms of failure loads.

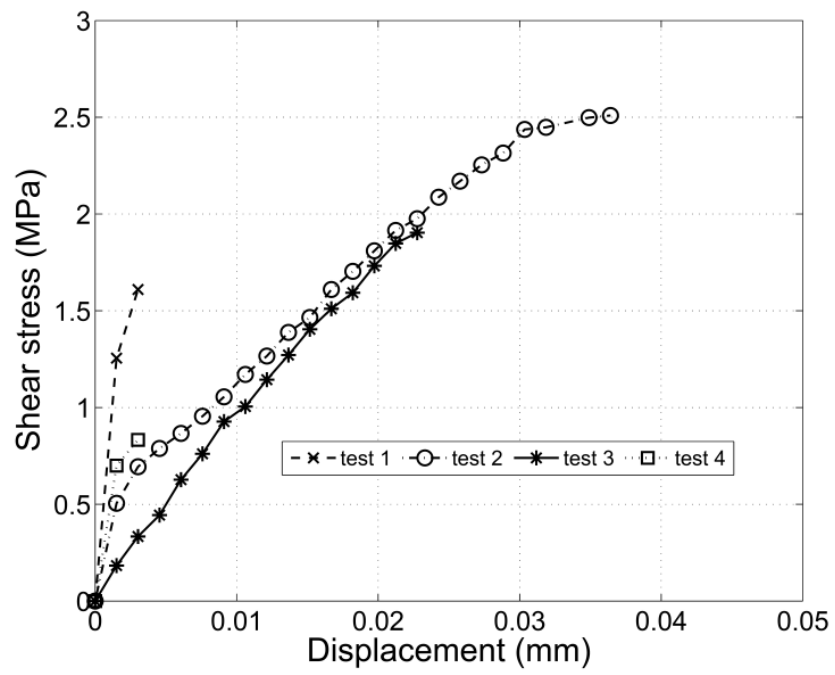

Fig. (4). Shear stress-displacement curve in the case of full brick triplets: experimental results (each curve represents a test).

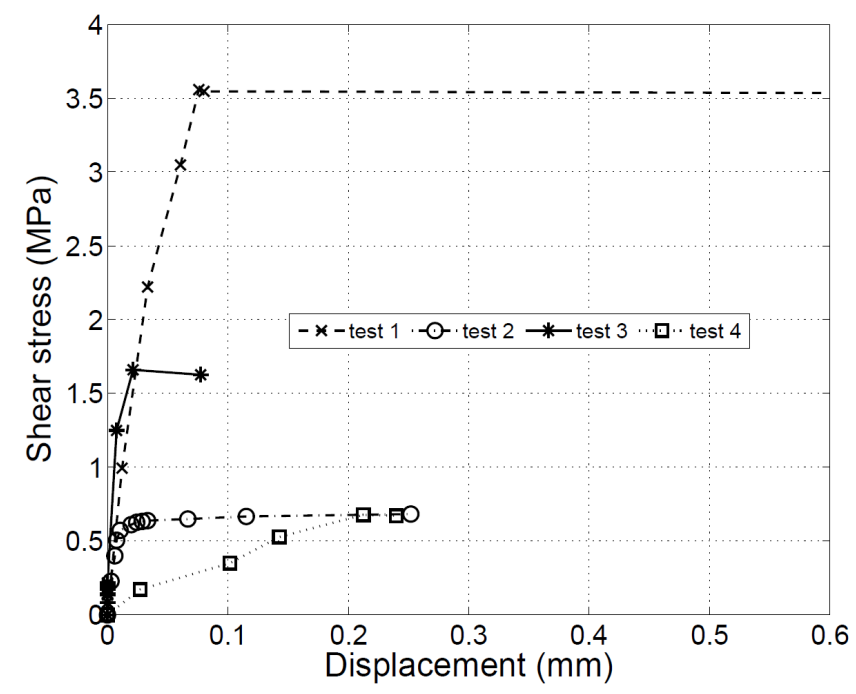

Fig. (5). Shear stress-displacement curve in the case of hollow brick triplets: experimental results (each curve represents a test).

Notably, two cracks localization are observed: at mortar/brick interface level and in the mortar joint. In both cases, i.e. full and hollow samples, proposed experimental evidence shows that the unit/mortar interface behavior is less brittle than the mortar one. Regarding the hollow bricks, other parameters can significantly affect the dispersion of the experimental data as the presence of an arbitrary distribution of mortar spikes in the brick hollows.

Finally, the main findings of this experimental part of the study are: the non-linear behaviour of masonry-like samples tested under shear conditions and the strong heterogeneity of this kind of structure at the local scale.

In previous works, it has been established that damaged interface models as RCCM model [10] and the Rekik-Lebon model $[13,14]$, are successfully able to reproduce the above experimental tests, which showed a sliding mode failure at the brick/mortar interface. 


\begin{tabular}{|c|c|c|c|c|c|}
\hline & & & & \multirow{2}{*}{ Test 3} & \multirow[b]{2}{*}{ Test 4} \\
\hline & & Test 1 & Test 2 & & \\
\hline \multirow{2}{*}{$\begin{array}{c}\text { Full } \\
\text { bricks }\end{array}$} & Failure modes & & & & \\
\hline & Shear stress (MPa) & 1.60 & 2.50 & 1.90 & 0.65 \\
\hline \multirow{2}{*}{$\begin{array}{l}\text { Hollow } \\
\text { bricks }\end{array}$} & Failure modes & & ": & & $\because$ \\
\hline & Shear stress (MPa) & 3,65 & 0.60 & 1.75 & 0,60 \\
\hline
\end{tabular}

Fig. (6). Failure modes experienced by testing triplets of full and hollow bricks, and corresponding ultimate shear stress values.

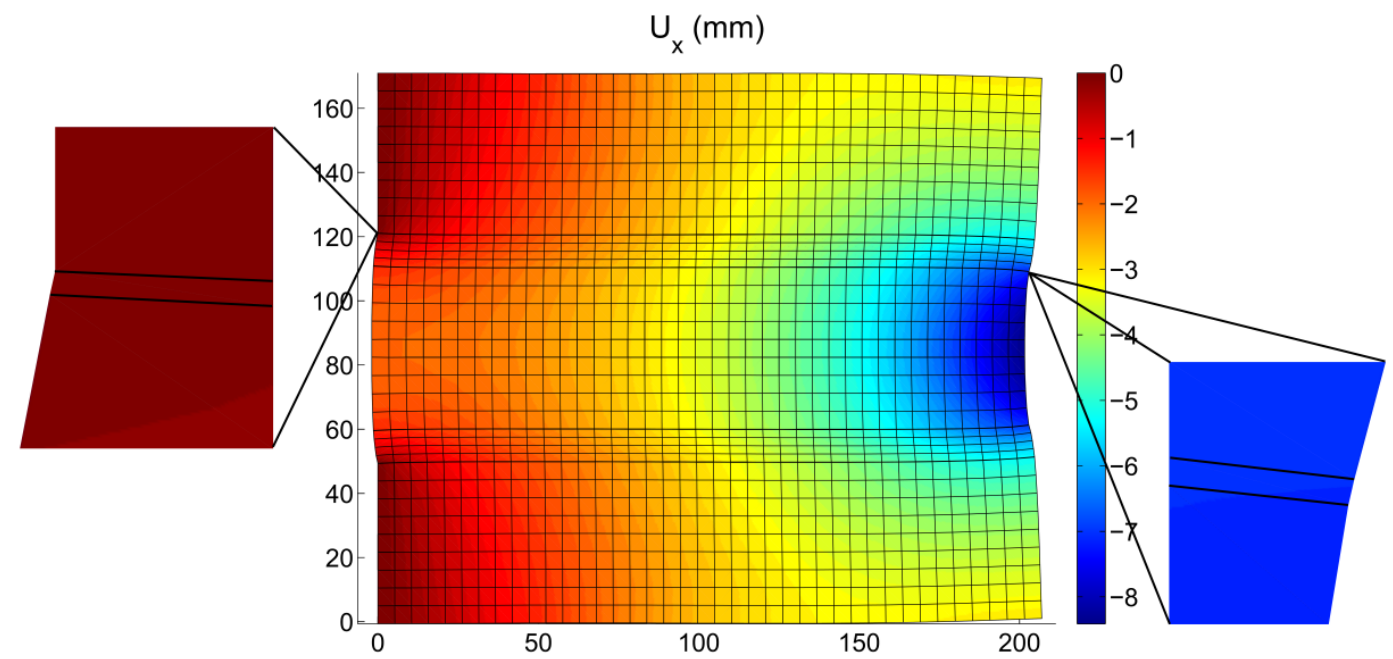

Fig. (7). Plot of the deformed shape and color map of the displacement in x-direction (obtained for a cosine roughness function $\eta$ with $A^{*}=0.5$ and $\left.T^{*}=2.1\right)$.

\section{NUMERICAL RESULTS AND DISCUSSION}

According to the Eqs. (20) and (21) of the interface model presented in Section 2, the interface stiffnesses in normal and tangential directions are expressed by simple closed-form relationships. Proposed numerical applications refer to the test 1 on the three full bricks sample, which exhibits a rupture localized at brick/mortar interface level (Fig. 4).

A finite element analysis is implemented. A plane stress modeling is pursued using a regular mesh of $Q 4$ elements for the overall computational domain. To reproduce brick/mortar interfaces, interface elements, based on the stiffness properties previously established, are used. An incremental explicit algorithm is used to solve the local problem [25]. For the constituents, material properties summarized in Table 1 is considered.

To show the influence of the roughness shape on the response of the masonry-like structural model, three different periodic functions, with the same period and amplitude, are considered: a cosine wave, a square wave and a sawtooth wave. For the sake of simplicity, these planar functions are chosen periodic in the plane $\left(\mathbf{i}_{1}, \mathbf{i}_{3}\right)$ and constant with respect to the coordinate $x_{2}$. In the following, these three different roughness functions will be indicated, respectively, as $\eta_{1}, \eta_{2}, \eta_{3}:$

$$
\left\{\begin{array}{l}
\eta_{1}=1+A^{*} \cos \left(\frac{2 \pi x_{1}}{T^{*}}\right) \\
\eta_{2}=1+A^{*} \operatorname{sgn}\left[\sin \left(\frac{2 \pi x_{1}}{T^{*}}\right)\right] \\
\eta_{3}=1+A^{*} \operatorname{frac}\left(\frac{x_{1}}{T^{*}}\right)
\end{array}\right.
$$

where, as customary, $\operatorname{frac}(x)=x-\lfloor x\rfloor$, and where the parameters $A^{*}=A / h$ and $T^{*}=T / h$ are, respectively, the amplitude and the period, normalized with respect to a characteristic dimension $h$, herein chosen as $h=10 \mathrm{~mm}$, i.e. equivalent to the mortar thickness. Dimensionless amplitude and period ( $A^{*}$ and $T^{*}$ ) are assumed to be equal, respectively, to 0.1 and 0.63 . It is worth pointing out that 
by introducing the roughness function $\eta$ within the interface constitutive law, the interface stiffnesses are no longer constant along the interface surface unlike in Rekik-Lebon model.

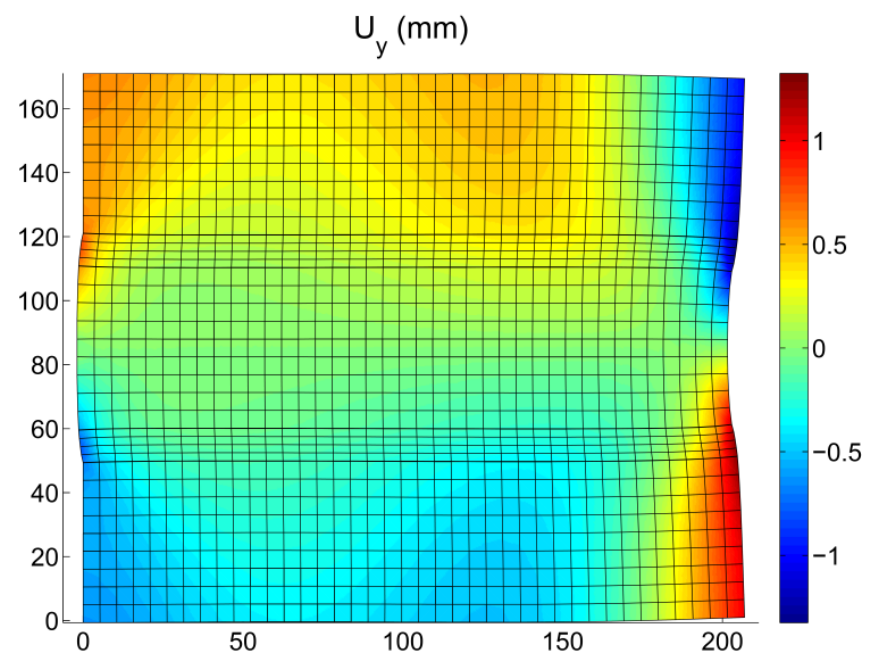

Fig. (8). Plot of the deformed shape and color map of the displacement in y-direction (obtained for a cosine roughness function $\eta$ with $A^{*}=0.5$ and $T^{*}=2.1$ ).

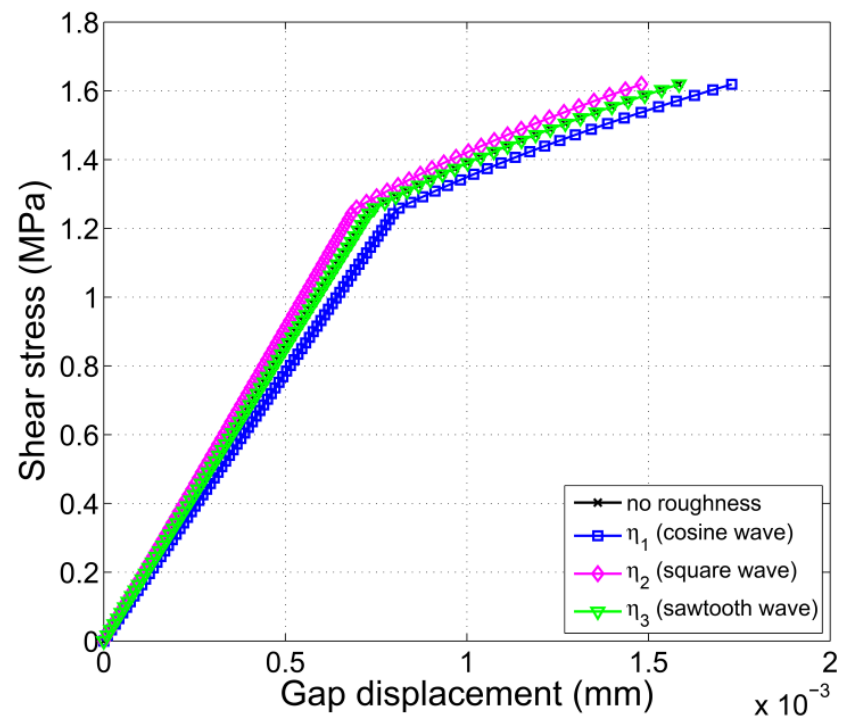

Fig. (9). Shear stress-displacement curve for a triplet model. Numerical results. Influence of roughness shape.

The numerical response of the triplet model in terms of "load-displacements" curves is obtained for the three types of roughness and in the case without roughness. Fig. (7) shows the influence of the shape of the roughness function for fixed amplitude and period. For the chosen values of these parameters, it is pointed out that the shape of the roughness does not have a relevant influence on the global response of the masonry-like model.

The cosine roughness function is chosen to make an identification process with respect to experimental results relevant to the test 1 of the triplets of full bricks. The period as well as the parameters of the bi-linear evolution law for $l$ as proposed in Rekik-Lebon [20], are assumed to be fixed
$\left(T^{*}=2.1, \tau_{c}=1.26 \mathrm{MPa}, \tau_{u}=1.62 \mathrm{MPa}, l_{c}=3.1 \mathrm{~mm}\right.$, $l_{u}=4.0 \mathrm{~mm}$ ), thus the proper amplitude value is identified as it is shown in Fig. (8).

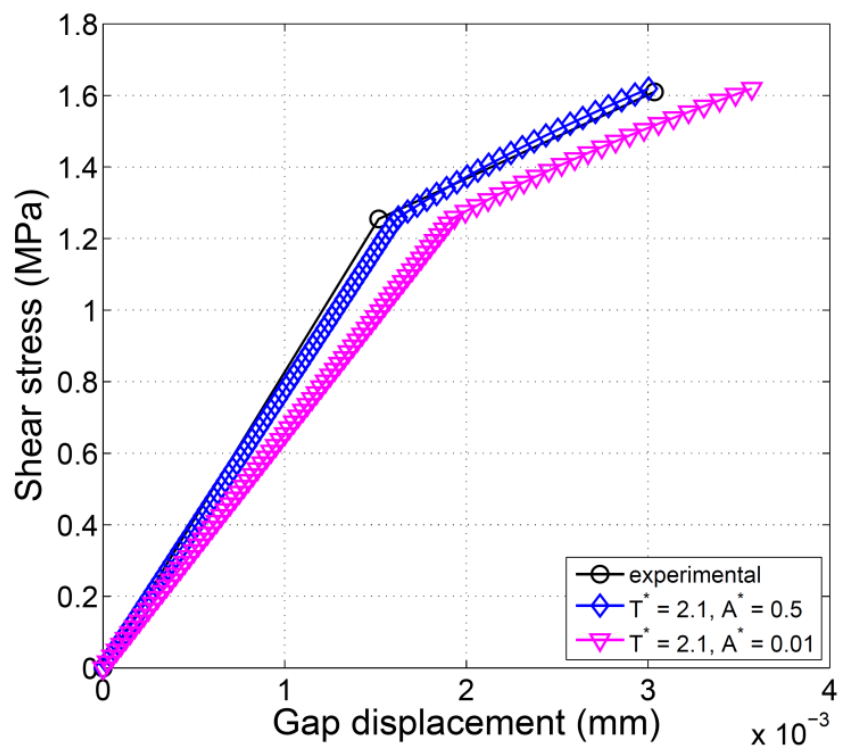

Fig. (10). Identification of the dimensionless amplitude of a cosineshaped roughness function, by comparison with experimental data.

Moreover, in order to understand the influence of roughness entity on the global shear stress-displacement response, a parametric analysis for the cosine-shaped roughness is performed by choosing different values of the normalized amplitude $A^{*}$ and period $T^{*}$, presented in Fig. (9) and Fig. (10).

As a general comment to these proposed numerical results, it is worth observing that the period $T^{*}$ does not have a great influence on the global response of the masonry-like model, for values of the dimensionless amplitude in the range $0 \leq A^{*} \leq 0.1$. Generally, for higher values of the normalized amplitude, namely $0.1 \leq A^{*}<1$, the influence of the normalized period seems to be much more relevant. Nevertheless, within the limits of the present approach and for any value herein considered for the period $T^{*}$, the amplitude $A^{*}$ can be retained as the roughness parameter that mainly affects the interface response.

\section{CONCLUSION}

In this paper a generalization of the interface model proposed by Rekik and Lebon has been established and applied to masonry-like structures. The model accounts for roughness and micro-cracks in the interface region, and it has been derived by employing an asymptotic expansions method. Numerical results obtained via the proposed formulation and based on a finite element implementation, have been successfully compared with experimental data relevant to samples of masonry assemblies. Within the limitation of the proposed approach, a parametric analysis has been carried out, highlighting the influence of the 

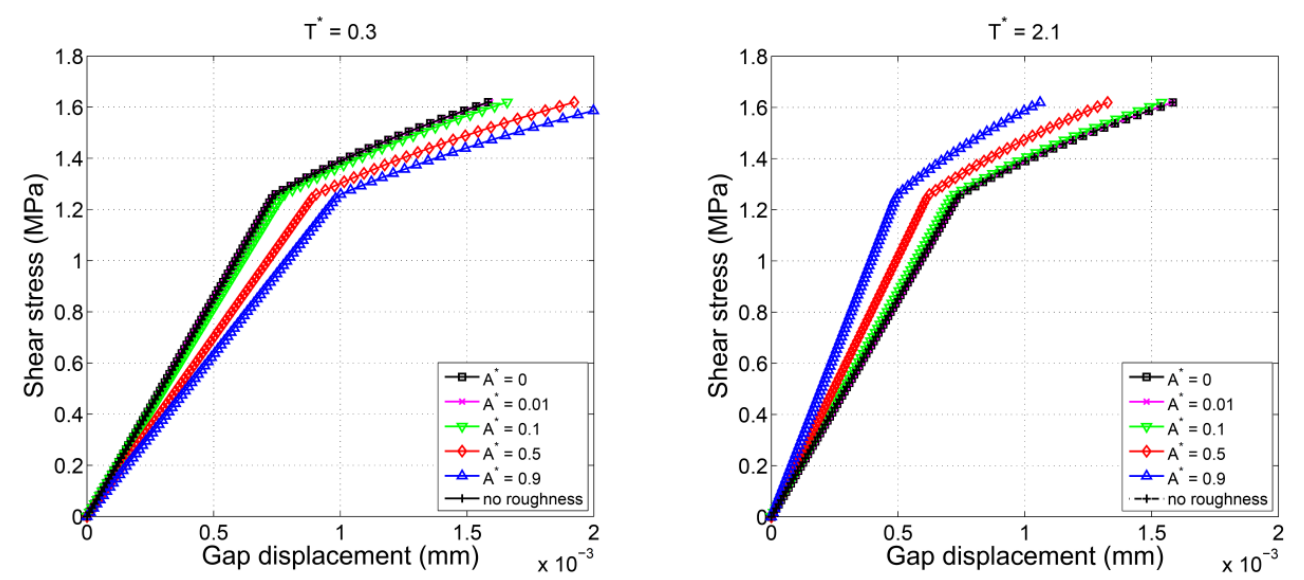

Fig. (11). Numerical results computed for a triplet model. Influence of the dimensionless amplitude of a cosine-shaped roughness function on the shear stress-displacement response.
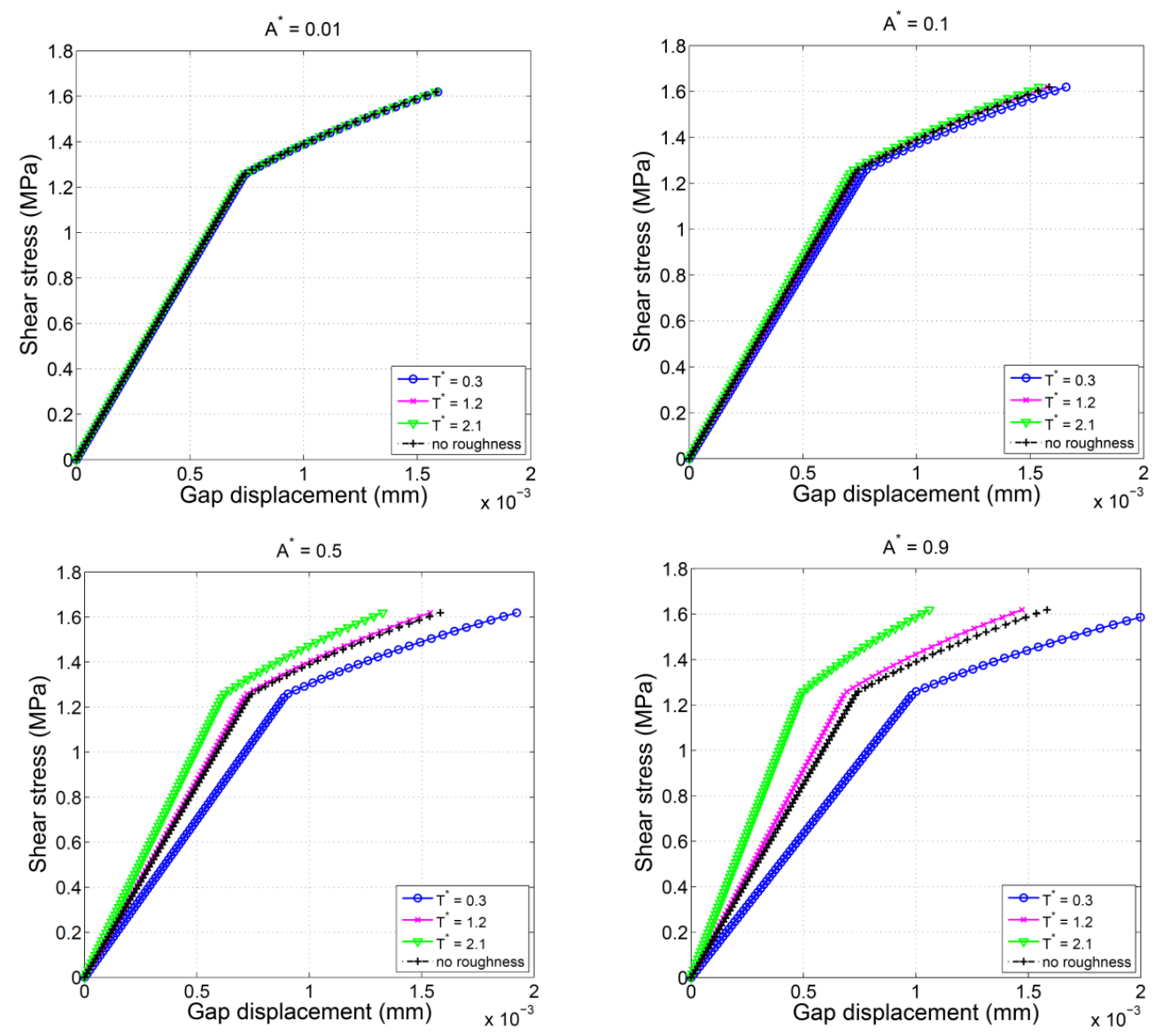

Fig. (12). Numerical results computed for a triplet model. Influence of the dimensionless period of a cosine-shaped roughness function on the shear stress-displacement response

roughness function features on the asymptotic constitutive behaviour of the interface.

Future research steps will be devoted to improve the micro-crack modeling, by including damage evolution descriptions and by enhancing the constitutive descriptions within the homogenization procedure, as proposed, for example, by Goidescu et al. [26]. Moreover, a comparison between the proposed model and well-established interface models is ongoing.

\section{CONFLICT OF INTEREST}

The authors confirm that this article content has no conflict of interest.

\section{ACKNOWLEDGEMENTS}

This work was supported by Bando Vinci 2013 (n. C273) of Università Italo-Francese, and by Italian Civil Protection Department (RELUIS-DPC 2014-16). 


\section{REFERENCES}

[1] A.W. Page, "Finite element model for masonry," J. Struct. Div., vol. 104, pp. 1267-1285, 1978.

[2] H.R. Lotfi and P.B. Shing, "Interface model applied to fracture of masonry structures," J. Struct. Eng. ASCE, vol. 120, no. 1, pp. 63$80,1994$.

[3] L. Gambarotta and S. Lagomarsino, "Damage models for the seismic response of brick masonry shear walls .1. the mortar joint model and its applications" Earthquake Eng. Struct. Dyn., vol. 26, no. 4, pp. 423-439, 1997.

[4] P.B. Lourenço and J.G. Rots, "Multisurface interface model for analysis of masonry structures," J. Struct. Eng. ASCE, vol. 123, no. 7, pp. 660-668, 1997.

[5] M. Raous, L. Cangemi, and M. Cocu, "A consistent model coupling adhesion, friction, and unilateral contact," Comp. Meth. App.Mech. Eng., vol. 177, no. 3-4, pp. 383-399, 1999.

[6] C. Calderini and S. Lagomarsino, "Continuum model for in-plane anisotropic inelastic behavior of masonry," J. Struct. Eng. ASCE, vol. 134, no. 2, pp. 209-220, 2008.

[7] A. Spada, G. Giambanco, and P. Rizzo, "Damage and plasticity at the interfaces in composite materials and structures," Comp. Meth. App.Mech. Eng., vol. 198, no. 49-52, pp. 3884-3901, 2009.

[8] Y. Monerie and M. Raous, "A model coupling adhesion to friction for the interaction between a crack and a fibre/matrix interface," Zeitschrift Fur Angewandte Mathematik Und Mechanik, vol. 80, pp. S205-S208, 2000.

[9] M. Frémond, “Adhesion of solids," J. De Mecanique Theorique Et Appliquee, vol. 6, no. 3, pp. 383-407, 1987.

[10] F. Fouchal, F. Lebon, and I. Titeux, "Contribution to the modelling of interfaces in masonry construction," Construct. Build. Mat., vol. 23, no. 6, pp. 2428-2441, 2009.

[11] G. Alfano and E. Sacco, "Combining interface damage and friction in a cohesivezone model," Int. J. Num. Methods Eng., vol. 68, no. 5, pp. 542-582, 2006.

[12] C. Pelissou and F. Lebon, "Asymptotic modeling of quasi-brittle interfaces,” Comp. Struct., vol. 87, no. 19-20, pp. 1216-1223, 2009.

[13] A. Rekik and F. Lebon, "Identification of the representative crack length evolution in a multi-level interface model for quasi-brittle masonry," Int. J. Solids Struct., vol. 47, no. 22-23, pp. 3011-3021, 2010.
[14] A. Rekik and F. Lebon, "Homogenization methods for interface modeling in damaged masonry," Adv. Eng. Software, vol. 46, no. 1, pp. 35-42, 2012.

[15] F. Lebon and R. Rizzoni, "Asymptotic behavior of a hard thin linear elastic interphase: An energy approach," Int. J. Solids Struct., vol. 48, no. 3-4, pp. 441-449, 2011.

[16] F. Lebon, R. Rizzoni, and S. Ronel-Idrissi, “Asymptotic analysis of some nonlinear soft thin layers," Comp. Struct., vol. 82, no. 23-26, pp. 1929- 1938, 2004.

[17] F. Lebon and F. Zaittouni, "Asymptotic modelling of interfaces taking contact conditions into account: Asymptotic expansions and numerical implementation," Int. J. Eng. Sci., vol. 48, no. 2, pp. 111-127, 2010 .

[18] I. Tsukrov and M. Kachanov, "Effective moduli of an anisotropic material with elliptical holes of arbitrary orientational distribution," Int. J. Solids Struct., vol. 37, no. 41, pp. 5919-5941, 2000.

[19] R. Rizzoni, S. Dumont, F. Lebon, and E. Sacco, "Higher order model for soft and hard interfaces," (submitted).

[20] F. Fouchal, "Contribution à la modélisation numérique des interfaces dans les structures maçonnées," Ph.D. dissertation, Reims University, 2006.

[21] E. Sanchez-Palencia, "Non-homogenous media and vibration theory," Springer- Verlag, 1980.

[22] F. Lebon, A. Ould Khaoua, and C. Licht, "Numerical study of soft adhesively bonded joints in finite elasticity," Comput. Mech., vol. 21, pp. 134-140, 1998

[23] R. v.d.Pluijm, "Out of plane bending of masonry behavior," $\mathrm{Ph} . \mathrm{D}$. dissertation, Eindhoven University of Technology, 1999.

[24] A. Gabor, "Contribution à la caractérisation et à la modélisation des maçonneries non-renforcées et renforcées par matériaux composites" Ph.D. dissertation, University Claude Bernard Lyon 1, 2002 .

[25] M.L. Raffa, F. Lebon, E. Sacco, and H.Welemane, "A multi-level interface model for damaged masonry," in B.H.V. Topping, P. Iványi, (Editors), "Proceedings of the Fourteenth International Conference on Civil, Structural and Environmental Engineering Computing", Civil-Comp Press, Stirlingshire, UK, Paper 64, 2013.

[26] C. Goidescu, H. Welemane, D. Kondo, and C. Gruescu, "Microcracks closure effects in initially orthotropic materials," Euro. J. Mech. A-Solids, vol. 37, pp. 172-184, 2013. 